\title{
The Magnetic Fields of Anomalous X-ray Pulsars
}

\author{
Feryal Özel*, Tolga Güver ${ }^{\dagger, *}$ and Ersin Göğüşs* \\ ${ }^{*}$ Department of Physics, University of Arizona, 1118. E. 4th St. Tucson, AZ 85704 \\ ${ }^{\dagger}$ Istanbul University, Science Faculty, Astronomy \& Space Sciences Department, Beyazlt, Istanbul, 34119 \\ ${ }^{* *}$ Sabancl University, Faculty of Engineering Natural Sciences, 34956 Turkey
}

\begin{abstract}
Anomalous X-ray Pulsars (AXPs) belong to a class of neutron stars believed to harbor the strongest magnetic fields in the universe, as indicated by their energetic bursts and their rapid spindowns. We have developed a theoretical model that takes into account processes in the atmospheres and magnetospheres of ultramagnetic neutron stars, as well as the effects of their strong gravitational fields on the observable properties. Using this model, we have analyzed the X-ray spectra of a number of AXPs. We find that in all cases, the X-ray spectra are described very well with this emission model. The spectroscopically measured magnetic field strengths of these sources are in close agreement with the values inferred from their spindown properties and provide independent evidence for their magnetar nature. The analysis of spectral data using this physical model also sheds light on the long-term evolution of AXPs.
\end{abstract}

Keywords: Pulsars, Magnetars

PACS: $97.60 . \mathrm{Gb}, 98.70 . \mathrm{Qy}$

\section{INTRODUCTION}

Anomalous X-ray Pulsars (AXPs) are thought to be the observational manifestations of a class of ultramagnetic $\left(B \gtrsim 10^{14} \mathrm{G}\right)$ neutron stars, also called magnetars (see Woods \& Thompson 2006 and Kaspi 2006 for reviews on magnetars and AXPs). Among the numerous spectral and timing properties of these isolated X-ray sources, two stand out for our focus in these proceedings. Their X-ray spectra are soft but non-Planckian, traditionally described by empirical functions such as a blackbody (kT $\sim 0.3-0.6 \mathrm{keV}$ ) plus a power law (with photon index $\Gamma \sim 2.5-4$ ) and, less frequently, by a sum of two blackbody functions (see, e.g., Gotthelf \& Halpern 2005; Kaspi 2006). The second property is their high spindown rates, with $\dot{P} \sim 10^{-11} \mathrm{~s} \mathrm{~s}^{-1}$.

A convincing, albeit indirect, argument for their strong magnetic fields arises from these large spindown rate 1 (e.g., Kouveliotou et al. 1998). Assuming the neutron stars spin down due to magnetic braking of a dipole in vacuum, their magnetic field strengths can be estimated by $B_{\text {dip }}=2.48 \times 10^{14}(P / 6 \mathrm{~s})^{1 / 2}\left(\dot{P} / 10^{-11} \mathrm{~s} \mathrm{~s}^{-1}\right)^{1 / 2} \mathrm{G}$, for a neutron star moment-of-inertia $I=10^{45} \mathrm{~g} \mathrm{~cm}^{2}$ and a neutron star radius of $R=10 \mathrm{~km}$. The dipole fields associated with AXPs thus exceed $B \gtrsim 5 \times 10^{13} \mathrm{G}$. The dipole spindown formula makes numerous assumptions when connecting period derivatives with a magnetic field

\footnotetext{
${ }^{1}$ The energetics and the timescales of intense, super-Eddington, random bursts of X-rays or soft gamma-rays seen in AXPs and the closely related Soft Gamma-ray Repeaters that last a fraction of a second also suggest independently the existence of very strong magnetic fields (Thompson \& Duncan 1995).
}

strength, such as a fiducial angle between the magnetic and rotation axes and the absence of other torques on the neutron star (Spitkovsky 2006). The dipole magnetic field inferred in this way has never been compared with an independent, spectroscopic measurement for an isolated pulsar.

Recently, there has been significant theoretical work on the emission from the atmospheres and magnetospheres of magnetars. As part of these efforts, we have developed a physical model of emission from a magnetar that takes into account processes in its atmosphere as well as in its magnetosphere. The Surface Thermal Emission and Magnetospheric Scattering (STEMS) model is based on the radiative equilibrium atmosphere calculations presented in Özel (2003) but also includes the effects of magnetospheric scattering of the surface radiation as discussed in Lyutikov \& Gavriil (2006) and Güver, Özel \& Lyutikov (2006). We also take into account the general relativistic effects in the strong gravitational field of the neutron star, making our models directly comparable to the wealth of spectral and timing data on AXPs. Naturally, comparison with such data is the ultimate test of any theoretical model. At the same time, a model that can describe consistently and in detail the spectra of AXPs can be used to understand the physical properties of these sources and their emission mechanisms.

In this proceedings paper, we present the results of applying the Surface Magnetospheric Scattering and Surface Emission Model to the soft X-ray data of four AXPs. In particular, we measure the magnetic field strength of these sources spectroscopically and we investigate the connection between the spectroscopically determined 
magnetic field strengths with those inferred from dipole spindown.

\section{THE THEORETICAL MODEL}

In highly magnetic, ionized neutron star atmospheres, polarization-mode dependent transport of radiation that includes absorption, emission, and scattering processes determines the continuum spectrum (see, e.g., Özel 2001, 2003). Furthermore, the interaction of the photons with the protons in the plasma gives rise to an absorption feature at the proton cyclotron energy $E_{p}=$ $6.3\left(B / 10^{15} \mathrm{G}\right) \mathrm{keV}$. This absorption feature is weakened by the vacuum polarization resonance, which also leads to an enhanced conversion between photons of different polarization modes as they propagate through the atmosphere.

In the magnetospheres of magnetars, currents supporting the ultrastrong magnetic fields can lead to enhanced charge densities (Thompson, Lyutikov, \& Kulkarni 2002), which reprocess the surface radiation through resonant cyclotron scattering (Lyutikov \& Gavriil 2006; Güver, Özel, \& Lyutikov 2007). We calculate this effect using the Green's function approach described in Lyutikov \& Gavriil (2006) assuming that the magnetosphere is spherically symmetric and the field strength follows a $1 / \mathrm{r}^{3}$ dependence.

In our spectral models, we include the relevant processes that take place on the magnetar surface and its magnetosphere, which depend only on four physical parameters. The first two parameters, the surface magnetic field strength $B$ and temperature $T$, describe the conditions found on the neutron star surface. The third parameter denotes the average energy of the charges $\beta=v_{e} / c$ in the magnetosphere, while the last parameter is related to the density $N_{e}$ of such charges and indicates the optical depth to resonant scattering by $\tau=\sigma \int N_{e} d z$. Here, $\sigma$ is the cross-section for resonant cyclotron scattering. We also assume a fixed value for the gravitational acceleration on the neutron star surface of $1.9 \times 10^{14} \mathrm{~cm} \mathrm{~s}^{-2}$, obtained for reasonable values of the neutron star mass and radius.

We calculated model X-ray spectra (in the $0.05-9.8$ $\mathrm{keV}$ range) by varying model parameters in suitable ranges that are in line with the physical processes we incorporated into the models: surface temperature $T=0.1$ to $0.6 \mathrm{keV}$, magnetic field $B=5 \times 10^{13}$ to $3 \times 10^{15} \mathrm{G}$, electron velocity $\beta=0.1$ to 0.5 , and optical depth in the magnetosphere $\tau=1$ to 10 . From the set of calculated spectra, we created a table model which we use within the X-ray spectral analysis package XSPEC (Arnaud 1996) to model the X-ray spectra of AXPs.

Our models predict strong deviations from a Planckian spectrum, with a hard excess that depends on the surface temperature as well as the magnetic field strength, and weak absorption lines due to the proton cyclotron resonance. Both the atmospheric processes and the magnetospheric scattering play a role in forming these spectral features and especially in reducing the equivalent widths of the cyclotron lines.

\section{ANALYSES OF AXP SPECTRA}

In this proceedings paper, we present the analysis of a total of four XMM-Newton observations of four AXPs. For 4U 0142+61, 1RXS J1708-4009, and XTE J1810-197 we chose the longest available $\mathrm{X}$-ray observation carried out by XMM or Chandra observatories (i.e., the observation with the highest total counts). For 1E 1048.1-5937, we used the longest observation of this source in quiescence. A longer observation taken just after a burst from this source will be presented elsewhere.

In Table 1, we present the list of the archival pointed X-ray observations of each source analyzed in this study. All of these observations were taken with the European Photon Imaging Camera (EPIC) PN camera. The observations of $1 \mathrm{E} 1048.1-5937$, 1RXS J1708-4009, and XTE J1810-197 were taken in the Small Window Mode, while the observation of $4 \mathrm{U} 0142+61$ was taken in the Fast Timing Mode.

The spectral analysis was performed using the XSPEC 11.3.2.t (Arnaud 1996). We assumed a fiducial gravitational redshift correction of 0.2 , which corresponds to a neutron star with mass $1.4 M_{\odot}$ and $R=13.8 \mathrm{~km}$. We calculate the fluxes for the $0.5-8.0 \mathrm{keV}$ energy range and quote errors for $90 \%$ confidence level.

\section{AXP 4U 0142+61}

$4 \mathrm{U} 0142+61$ is the brightest known AXP and has historically been very stable. Rotating with a $8.69 \mathrm{~s}$ period (Israel et al. 1994), it spins down at a rate of $\dot{P} \approx 0.196 \mathrm{~s} \mathrm{~s}^{-1}$, yielding a $B_{\text {dip }}=1.3 \times 10^{14} \mathrm{G}$ using the dipole spindown formula. Multiple X-ray observations of the source showed a long epoch of nearly constant flux levels as well as a relatively hard X-ray spectrum (Juett et al. 2002; Patel et al. 2003; Göhler, Wilms \& Staubert 2005). Recently, the source exhibited SGR like bursts (Kaspi, Dib \& Gavriil 2006; Dib et al. 2006; Gavriil et al. 2007) for the first time.

$4 \mathrm{U} 0142+61$ has also been detected in hard X-rays with INTEGRAL (Kuiper et al. 2006, den Hartog et al. 2007a). The hard X-ray spectral component in the $20-230 \mathrm{keV}$ energy range is well described by a power law model of index 0.79 and the corresponding flux 
TABLE 1. Observations used for this study.

\begin{tabular}{ccccccc}
\hline Source & Satellite & Detector & Mode & $\begin{array}{c}\text { Exposure } \\
\text { Time (ks) }\end{array}$ & Obs ID & Obs Date \\
\hline 4U 0142+61 & XMM-Newton & EPIC-PN & Fast Timing & 21.1 & 0206670101 & Jul 25 2004 \\
1E 1048.1-5937 & XMM-Newton & EPIC-PN & Small Window & 32.44 & 0307410201 & Jun 16 2005 \\
1RXS J1708-4009 & XMM-Newton & EPIC-PN & Small Window & 44.9 & 0148690101 & Aug 29 2003 \\
XTE J1810-197 & XMM-Newton & EPIC-PN & Small Window & 42.2 & 0301270501 & Mar 18 2005 \\
\hline
\end{tabular}

is $1.7 \times 10^{-10} \mathrm{erg} \mathrm{cm}^{-2} \mathrm{~s}^{-1}$ (den Hartog et al. 2007a), which exceeds by a factor of $\sim 2$ the unabsorbed 2-10 $\mathrm{keV}$ flux. If this component extends without a break towards lower photon energies it contributes significantly to the soft X-ray flux in the $7-10 \mathrm{keV}$ range. Because of this, in our present analysis, we take into account the effect of this component by using the fits to the hard Xray observations reported by den Hartog et al. (2007a), assuming that this component extends to the soft X-rays without a break.

\section{E 1048.1-5937}

Several properties distinguish 1E 1048.1-5937 from the other AXPs. An ongoing RXTE monitoring campaign (Gavriil and Kaspi 2004) revealed that it shows long-lived pulsed flux flares in addition to SGR-like bursts. The spindown of the $6.452 \mathrm{~s}$ period pulsar is very unstable, with period derivative values in the range $\dot{P}=$ $0.8546(50)-3.81 \times 10^{-11} \mathrm{~s} \mathrm{~s}^{-1}$ (Kaspi et al. 2001). This yields a large range of dipole magnetic field strengths estimated from spindown. As a conservative range, we will adopt $B_{\text {dip }}=2.4-4 \times 10^{14} \mathrm{G}$ for this source.

1E 1048.1-5937 has been observed by the Chandra and XMM observatories as part of ongoing campaigns to monitor the variability of this AXP. The longest observation to date was taken by XMM on 16 June 2003, shortly after bursting activity. To focus on the quiescent properties of this source, as with the other AXPs, we analyze here the longest observation in quiescence, taken on 16 June 2005.

\section{RXS J1708-4009}

1RXS J1708-4009 is an $11.0 \mathrm{~s}$ AXP, initially thought to be a fairly stable rotator (Israel et al. 1999). In the last several years, the source experienced multiple glitches (e.g., Dib et al. 2007) that interrupted stretches of steady spin-down. A period derivative of $\dot{P} \approx 1.4-$ $1.9 \times 10^{-11} \mathrm{~s} \mathrm{~s}^{-1}$ yields a dipole magnetic field strength of $B_{\text {dip }}=4.0-4.7 \times 10^{14} \mathrm{G}$.

As in the case of $4 \mathrm{U} 0142+61$, 1 RXS J1708-4009 exhibits a hard, pulsed hard X-ray tail extending to energies up to $\sim 150 \mathrm{keV}$ (Kuiper et al. 2006). Here we adopt the values given by den Hartog et al. (2007b) with $\Gamma=1.17$ and the $20-250 \mathrm{keV}$ flux $6.2 \times 10^{-11} \mathrm{erg} \mathrm{cm}^{-2} \mathrm{~s}^{-1}$.

\section{XTE J1810-197}

In the opposite extreme from $4 \mathrm{U} \quad 0142+61$, XTE J1810-197 is the most variable confirmed AXP observed to date. It was discovered (Ibrahim et al. 2004) in 2003 when it suddenly brightened to more than 100 times its quiescent value (Halpern \& Gotthelf 2005) during an outburst. The source showed a steady decline of its X-ray flux thereafter, down to unusually low quiescent flux levels that have been determined from archival XTE and ROSAT data, accompanied by significant spectral changes (Gotthelf \& Halpern 2006), earning it the title of the transient AXP. The detection of characteristic X-ray bursts (Woods et al. 2005), similar to those seen in other AXPs (Gavriil, Kaspi, \& Woods 2002), further strengthen its classification as an AXP.

XTE J1810-197 has a $5.54 \mathrm{~s}$ period, and an unsteady spindown characterized by a $\dot{P} \approx 10^{-11} \mathrm{~s} \mathrm{~s}^{-1}$ period derivative measured in the X-rays (Ibrahim et al. 2004; Gotthelf \& Halpern 2005). The detection of radio emission from the source, first ever for an AXP (Camilo et al. 2006), allowed for a more closely-spaced monitoring of its period and resulted in the measurement of a larger range of spindown rates (Camilo et al. 2007). The range of $B_{\text {dip }}$ corresponding to the observed period derivatives are used in Figure 5.

In an earlier investigation, we reported on the physical evolution of this source during its decline from outburst (Güver et al. 2007). Here, we focus on the magnetic field strength of XTE J1810-197 using the observation with the highest number of counts.

\section{DISCUSSION}

Figure 5 shows the magnetic field strengths obtained for the 4 AXPs by fitting their X-ray spectra with the STEMS model against the dipole field strengths inferred from the spindown of these sources according to the dipole spindown formula. The error bars correspond to 
TABLE 2. Spectral Results of STEMS Model for 4 AXPs

\begin{tabular}{cccccc}
\hline Source & Magnetic Field & Surface Temperature & $\tau$ & $\beta$ & $\chi_{v}^{2}$ (d.o.f.) \\
\hline 4U 0142+61 & $4.60 \pm 0.07$ & $0.31 \pm 0.01$ & $3.54 \pm 0.14$ & $0.43 \pm 0.01$ & $0.931(462)$ \\
1E 1048.1-5937 & $2.26 \pm 0.05$ & $0.37 \pm 0.01$ & $3.91 \pm 0.52$ & $0.22 \pm 0.02$ & $0.952(611)$ \\
1RXS J1708-4009 & $3.96 \pm 0.17$ & $0.35 \pm 0.01$ & $5.26 \pm 0.36$ & $0.48 \pm 0.01$ & $1.050(1187)$ \\
XTE J1810-197 & $2.68 \pm 0.06$ & $0.31 \pm 0.01$ & $2.36 \pm 0.36$ & $0.25 \pm 0.02$ & $1.07(732)$ \\
\hline
\end{tabular}

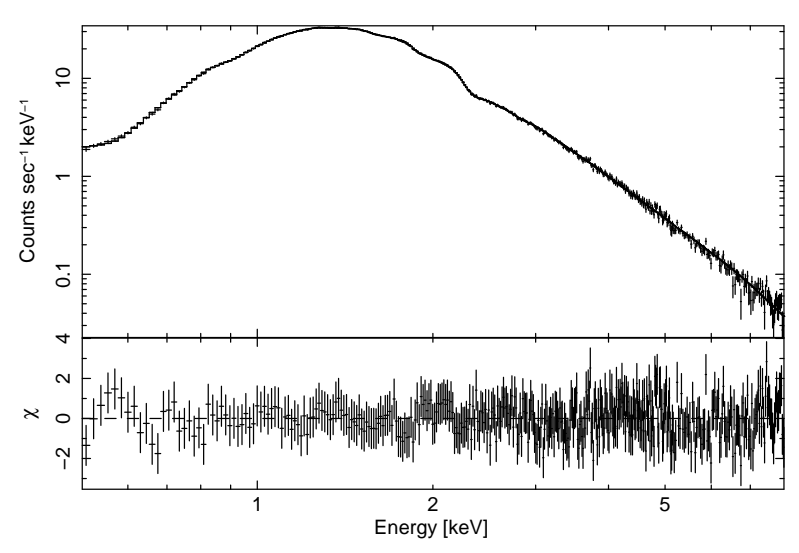

FIGURE 1. STEMS model fit to the X-ray spectrum of 4U 0142+61.

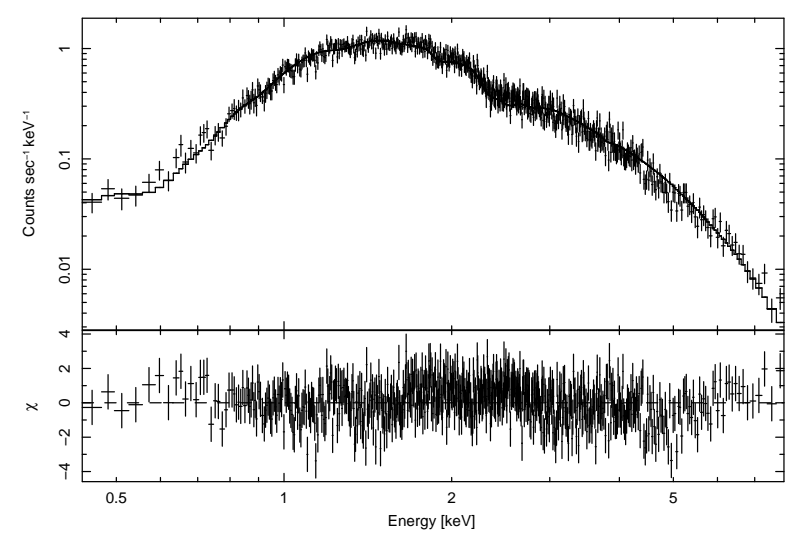

FIGURE 2. STEMS model fit to the X-ray spectrum of 1E 1048.1-5937.

$2-\sigma$ uncertainty in the values of the spectroscopic magnetic field strength, while error bars on the dipole spindown field reflect the range obtained from the variable period derivatives seen in some sources.

In the cases of 1E 1048.1-5937, 1RXS J1708-4009, and XTE J1810-197, we find a very good agreement, at an unexpected level, between the spectroscopically measured magnetic field strength and that obtained from their spindown. In all three cases, the two values are consistent within the formal and expected systematic uncertainties. For the case of $4 \mathrm{U} 0142+61$, the spectroscopically measured surface magnetic field is a factor of 3 larger than

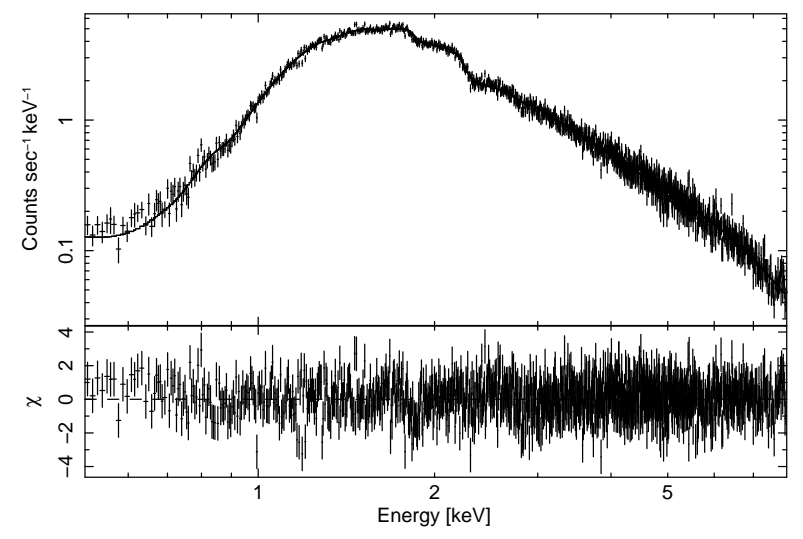

FIGURE 3. STEMS model fit to the X-ray spectrum of RXS J1708-4009.

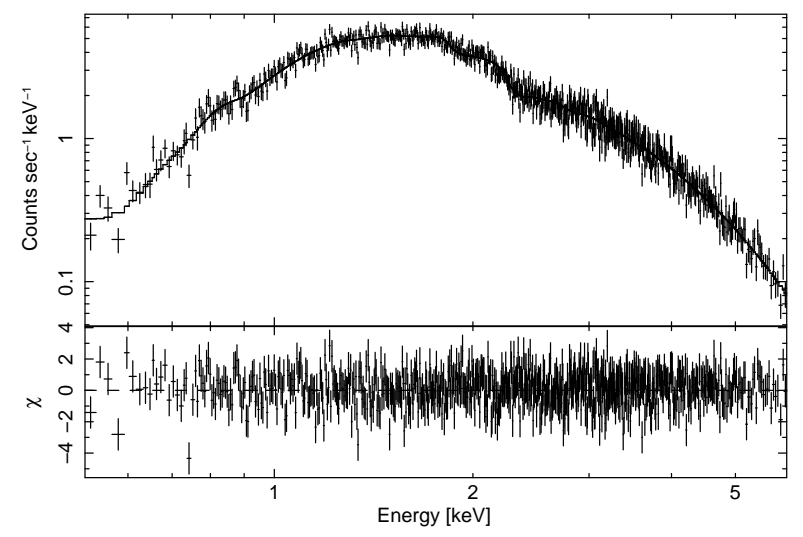

FIGURE 4. STEMS model fit to the X-ray spectrum of XTE J1810-197.

the spindown field. This may be due to the simplified assumptions in either of the two measurements of the magnetic field. Alternatively, this might be an indication of multipole magnetic field components on the neutron star surface which contribute negligibly to the spindown torques.

The good agreement between the theoretical models and the spectral data provide us with a new tool with which to understand the physical conditions of magnetar surfaces and magnetospheres. At the same time, our spectroscopic measurements of the magnetic field strengths offer independent confirmation for the magne- 


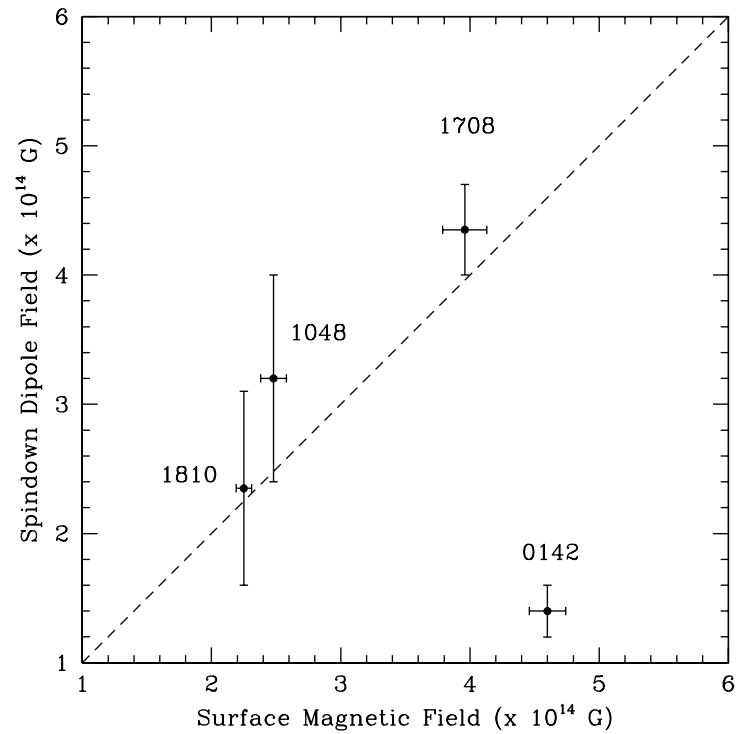

FIGURE 5. The comparison of the spectroscopically measured magnetic field strengths of five AXPs to the dipole fields inferred from the spindown properties of these sources. The error bars in $B_{\text {dip }}$ represent the range of measured spindown rates for each source, while the error bars in the spectroscopic magnetic field strength represent $2-\sigma$ statistical uncertainties.

tar nature of AXPs.

\section{ACKNOWLEDGMENTS}

F.O. acknowledges support from NSF grant AST-0708640. We thank the McGill Pulsar group, and in particular, C. Tam, for maintaining the online AXP/SGR catalog http://www.physics.mcgill.ca/ pulsar/magnt that was helpful in preparing this publication.

\section{REFERENCES}

1. Arnaud, K.A., Astronomical Data Analysis Software and Systems $V$, edited by Jacoby G. and Barnes J., ASP Conference Series, 101, p17 (1996).

2. Camilo, F., et al., Nature, 442, 892-895 (2006).

3. Camilo, F., et al., The Astrophysical Journal, 669, 561-569 (2007).

4. den Hartog, P.R., et al., Astrophysics and Space Science, 308, 647-653 (2007).

5. den Hartog, P. R., Kuiper, L., Hermsen, W., Kaspi, V.M., Dib., R., Knoedesleder, J., This Volume, (2007).

6. Dib, R., Kaspi, V.M., Gavriil, F.P., Woods, P.M., The Astronomer's Telegram, 845, 1 (2006).

7. Dib, R., Kaspi, V. M., Gavriil, F. P., accepted by The Astrophysical Journal, arXiv:0706.4156 2 [astro-ph],
(2007).

8. Gavriil, F. P., Kaspi, V. M., Woods, P. M., Nature, 419, 142-144 (2002).

9. Gavriil, F.P., Kaspi, V.M., The Astrophysical Journal Letters, 609, 67-70 (2004).

10. Gavriil, F.P., Dib, R., Kaspi, V.M., Woods, P.M., The Astronomer's Telegram, 993, 1 (2007).

11. Gotthelf, E. V., Halpern, J. P., The Astrophysical Journal, 632, 1075-1085 (2005).

12. Gotthelf, E. V., Halpern, J. P., Astrophysics and Space Science, 308, 79-87 (2007).

13. Göhler, E., Wilms, J. \& Staubert, R., Astronomy \& Astrophysics, 433, 1079-1083 (2005).

14. Güver, T., Özel, F., Lyutikov, M., submitted to The Astrophysical Journal, arXiv:astro-ph/0611405 1 , (2006).

15. Güver, T., Özel, F., Göğüss, E., Kouveliotou, C. The Astrophysical Journal Letters, 667, 73-76 (2007).

16. Ibrahim, A., et al., The Astrophysical Journal Letters, 609, 21-24 (2004).

17. Israel, G. L., Mereghetti, S., Stella, L., The Astrophysical Journal Letters, 433, 25-28 (1994).

18. Israel, G. L., Covino, S., Stella, L., Campana, S., Haberl, F., Mereghetti, S., The Astrophysical Journal Letters, $\mathbf{5 1 8}$ 107-110 (1999).

19. Juett, A.M., Marshall, H.L., Chakrabarty, D., Schulz, N.S., The Astrophysical Journal Letters, 568, 31-34 (2002).

20. Kaspi, V.M., Gavriil, F.P., Chakrabarty, D., Lackey, J.R., Muno, M.P., The Astrophysical Journal, 558, 253-262 (2001).

21. Kaspi, V.M., Dib, R., Gavriil, F., The Astronomer's Telegram, 794, 1 (2006).

22. Kaspi, V. M., Astrophysics \& Space Science, 308, 1 (2007).

23. Kouveliotou, C., et al., Nature, 393, 235-237 (1998).

24. Kuiper L., Hermsen W., den Hartog P. R., Collmar, W., The Astrophysical Journal, 645, 556-575 (2006).

25. Lyutikov, M., \& Gavriil, F.P., Monthly Notices of Royal Astronomical Society, 368, 690-706 (2006).

26. Özel, F., The Astrophysical Journal, 563, 276-288 (2001).

27. Özel, F., The Astrophysical Journal, 583, 402-409 (2003). gnet datems.iK., bttah.] The Astrophysical Journal, 587, 367-372 (2003).

29. Spitkovsky, A., Astrophysical Journal Letters, 648, L51 (2006).

30. Thompson, C., Duncan, R.C., Monthly Notices of Royal Astronomical Society, 275, 255-300 (1995).

31. Thompson C., Lyutikov M., Kulkarni S.R., The Astrophysical Journal, 574, 332-355 (2002).

32. Woods, P.M., et al., The Astrophysical Journal, 629 , 985-997 (2005).

33. Woods, P.M., Thompson, C., "Soft gamma repeaters and anomalous X-ray pulsars: magnetar candidates," in Compact stellar X-ray sources, edited by Walter Lewin $\&$ Michiel van der Klis., Cambridge University Press, Cambridge, 2006, pp. 547-586 